\title{
Measurement of food colour in L*a*b* units from RGB digital image using least squares support vector machine regression
}

\author{
Roberto Romaniello, Alessandro Leone, Giorgio Peri \\ Department of the Sciences of Agriculture, Food and Environment, University of Foggia, \\ Italy
}

\begin{abstract}
The aim of this work is to evaluate the potential of least squares support vector machine (LS-SVM) regression to develop an efficient method to measure the colour of food materials in $\mathrm{L}^{*} \mathrm{a}^{*} \mathrm{~b}^{*}$ units by means of a computer vision systems (CVS). A laboratory CVS, based on colour digital camera (CDC), was implemented and three LS-SVM models were trained and validated, one for each output variables ( $\mathrm{L}^{*}$, $\mathrm{a}^{*}$, and $\mathrm{b}^{*}$ ) required by this problem, using the RGB signals generated by the CDC as input variables to these models. The colour target-based approach was used to camera characterization and a standard reference target of 242 colour samples was acquired using the CVS and a colorimeter. This data set was split in two sets of equal sizes, for training and validating the LS-SVM models. An effective two-stage grid search process on the parameters space was performed in MATLAB to tune the regularization parameters $\gamma$ and the kernel parameters $\sigma^{2}$ of the three LS-SVM models. A 3-8-3 multilayer feed-forward neural network (MFNN), according to the research conducted by León et al. (2006), was also trained in order to compare its performance with those of LS-SVM models. The LS-SVM models developed in this research have been shown better generalization capability then the MFNN, allowed to obtain high correlations between $\mathrm{L}^{*} \mathrm{a}^{*} \mathrm{~b}^{*}$ data acquired using the colorimeter and the corresponding data obtained by transformation of the RGB data acquired by the CVS. In particular, for the validation set, $\mathrm{R}^{2}$ values equal to $0.9989,0.9987$, and 0.9994 for $\mathrm{L}^{*}$, $\mathrm{a}^{*}$ and $\mathrm{b}^{*}$ parameters were obtained. The root mean square error values were $0.6443,0.3226$, and 0.2702 for $\mathrm{L}^{*}, \mathrm{a}^{*}$, and $\mathrm{b}^{*}$ respectively, and the average of colour differences $\Delta \mathrm{E}_{\mathrm{ab}}$ was $0.8232 \pm 0.5033$ units. Thus,
\end{abstract}

Correspondence: Roberto Romaniello, Department of the Sciences of Agriculture, Food and Environment, University of Foggia, via Napoli 25, 71122 Foggia, Italy.

Tel.: +39.0881.589.120.

E-mail: roberto.romaniello@unifg.it

Key words: Food colour; CIELab colour space; colour measurements; digital camera.

Received for publication: 10 June 2015

Accepted for publication: 14 October 2015.

(C) Copyright R. Romaniello et al., 2015

Licensee PAGEPress, Italy

Journal of Agricultural Engineering 2015; XLVI:482

doi:10.4081/jae.2015.482

This article is distributed under the terms of the Creative Commons Attribution Noncommercial License (by-nc 3.0) which permits any noncommercial use, distribution, and reproduction in any medium, provided the original author(s) and source are credited.
LS-SVM regression seems to be a useful tool to measurement of food colour using a low cost CVS.

\section{Introduction}

In food quality monitoring, colour is an important quality indicator. The CIELab ( $\left.\mathrm{L}^{*} \mathrm{a}^{*} \mathrm{~b}^{*}\right)$ colour space, as a device independent colour space is an appropriate means in this case.

Usually the colour of foodstuff is detected by instruments called colour-meters and measured in device-independent colour space, i.e., CIELab ( $\left.\mathrm{L}^{*} \mathrm{a}^{*} \mathrm{~b}^{*}\right)$. The colorimeter permits the colour measurement only in a contact-mode and for a small area of the sample. However, developing algorithms for conversion of foodstuff RGB images into $\mathrm{L}^{*} \mathrm{a}^{*} \mathrm{~b}^{*}$ colour space can solve both of these issues (Sharifzadeh et al., 2014).

In recent years, computer vision systems (CVS) based on colour digital cameras (CDC) have been shown to be a good tool to quantify easily and quickly the colour of any food, using equipment that is readily available at reasonable cost (Mendoza et al., 2006). However, there are some issues that are important to consider for colour measurements. Specifically, the RGB signals generated by a colour digital camera are device-dependent, and these colour signals are not colorimetric. Therefore, in order to objectively measure colour and detect colour features from food products with accuracy, a colorimetric transformation that defines a mapping between the device-dependent RGB signals and a device-independent colour space, such as the $\mathrm{L}^{*} \mathrm{a}^{*} \mathrm{~b}^{*}$ (or CIELab) colour space, is an essential step in the implementation of any computer vision system (Valous et al., 2009).

Recently, a multilayer feed-forward neural network (MFNN) with back-propagation was used to defines a mapping between the RGB values generated by a CDC and the $\mathrm{L}^{*} \mathrm{a}^{*} \mathrm{~b}^{*}$ values with good results (León et al., 2006). However, MFNN suffer critical drawbacks including learning stopping at local minima, over-fitting, and selection type depending excessively on own experience (Wang et al., 2009).

Support vector machines (SVM), a new learning algorithm based on the statistical learning theory, can model linear and nonlinear mappings without these disadvantages (Vapnik, 2000). Unlike the classical neural networks approach the SVM formulation of the learning problem leads to quadratic programming (QP) with linear constraint. However, the size of matrix involved in the QP problem is directly proportional to the number of training points. Hence, to reduce the complexity of optimization processes, a modified version, called least squares support vector machines (LS-SVM), has proposed (Suykens and Vanderwalle, 1999). LS-SVM encompass similar advantage as SVM, but its additional advantage is that it requires solving a set of only linear equations (linear programming) that is much easier and computational more simple. For this reason, the application of LS-SVM resulted simpler than MFNN. Recently LS-SVM was employed to develop an automatic adjustable algorithm for segmentation of colour 
images, using linear support vector machine (SVM) and, for apple sorting and grading (Mizushima and Lu, 2013).

In this study, after the implementation of a laboratory CVS based on a CDC, the objective is to evaluate the potential of LS-SVM regression to solve the camera characterization problem. For this purpose, three LS-SVM models were trained and validated, one for each output variables ( $\mathrm{L}^{*}, \mathrm{a}^{*}$, and $\mathrm{b}^{*}$ ), using the RGB signals generated by the CDC as input variables to these models. An applicative case was carried out in order to test the best model on fruit colour measurement.

\section{Materials and methods}

\section{Computer vision system}

The implemented CVS has three main components: an illumination source, a CDC and an image processing software. The light source consisted of four fluorescent $15 \mathrm{~W}$ lamps (Neon OSRAM TLD65-15W; OSRAM GmbH, Munich, Germany) with a colour temperature of $6500 \mathrm{~K}$, arranged in a square $0.68 \mathrm{~m}$ above the sample. To ensure uniform illumination, the four lamps were connected to electronic ballasts and covered with plastic light diffusers.

The colour digital camera was a Canon EOS 400D (Canon USA, Inc., Melville, NY, USA) located vertically over the matte black background at a distance of $0.45 \mathrm{~m}$. The camera was connected to the USB port of a PC (Asus Computer Inc., Taipei, Taiwan) with a Remote Capture Software (version 2.7.2, Canon USA, Inc.) to visualize and receive the digitized images directly from the computer.

As standard setting conditions, the viewing/illuminating geometry was about $0 / 45$. Moreover, the sample illuminators and the camera were placed inside a wooden box with black internal surfaces to exclude external light and reflection. A standard white card (X-rite ColourChecher ${ }^{\circledR}$ White balance Card; X-Rite Inc., Grand Rapids, MI, USA) was used to set manually the white balance of the CDC (Figure 1A). Spatial correction was performed in order to minimise the effect of any lack of spatial uniformity in the intensity of the illumination or of the sensitivity of the CDC. The spatial correction method was based on work by Westland et al., 2004. Manual exposure mode and both lens aperture $(f=6.3)$ and exposure time $(1 / 4)$ where fixed during the period of image acquisition. In this experimentation, all images were acquired with resolution of $3888 \times 2592$ pixels (corresponding to a field of view of $32.4 \times 21.6 \mathrm{~cm}^{2}$ ), and stored in TIFF format. The image processing software was performed using the MATLAB v7.0 (The MathWorks, Natick, MA, USA) image processing toolbox.

\section{Reference target}

A practical method to camera characterization, referred as to colour target-based approach, was used. The basic idea of colour target-based characterization is to use a reference target that contains a certain number of colour samples of known CIE (Commission Internationale de l'Eclairage) values, which are contrasted with the output average signals captured in standard illumination conditions by an imaging sensor (Hong et al., 2001). In this study, the reference target was the Kodak Q60R2 reflection chart on Kodak Professional paper (Eastman Kodak Company, Rochester, NY, USA). This chart is manufactured in accordance with ANSI IT8.7/2- 1993 and ISO 12641 standards and it provides 264 colour samples, including a 22 step neutral scale, which cover a large gamut in the CIELab colour space (Figure 1B).

\section{Colour measurements}

For colour measurements, the reference target, located in the centre of the camera field view, was imaged to obtain for each colour samples the camera RGB values in the theoretical range 0-255. The camera RGB values for each colour sample were measured using a MATLAB program, which computes the average RGB values of $80 \%$ of the pixels in the samples, excluding the boundary pixels. Then, the $\mathrm{L}^{*} \mathrm{a}^{*} \mathrm{~b}^{*}$ values of each colour samples (D65 illuminant and $2^{\circ}$ observer) were measured using a colorimeter (CM-2600d, KONICA MINOLTA SENSING Inc., Osaka, Japan). This instrument measure spectral reflectance from 380 $700 \mathrm{~nm}$ in $20 \mathrm{~nm}$ intervals from a circular area of $3 \mathrm{~mm}$ diameter using a silicon photodiode and a diffraction grating device. The specular component included mode was set and the white reference was the white calibration plate of the colorimeter $\left(\mathrm{L}^{*}=99.30, \mathrm{a}^{*}=-0.09, \mathrm{~b}^{*}=-0.17\right)$. In total, a data set of 264 RGB measurements and their corresponding $\mathrm{L}^{*} \mathrm{a}^{*} \mathrm{~b}^{*}$ measurements were obtained from the CVS and the colorimeter respectively. This data set was split in two sets of equal sizes, for training and validating the LS-SVM models.

\section{Food colour measurement}

For food colour measurement, four species of fruit, with different dominant hue, were chosen: lime, apricot, red peach, yellow apple. For each kind of specie, three unities ware considered. A portion of skin has been cut with a circular blade, having area of about $1 \mathrm{~cm}^{2}$. The cir-

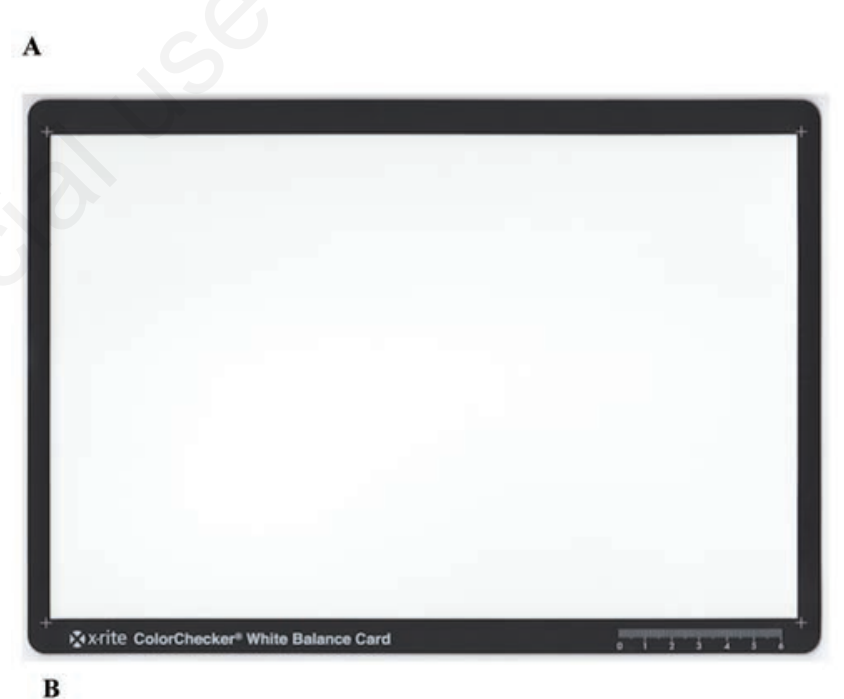

B

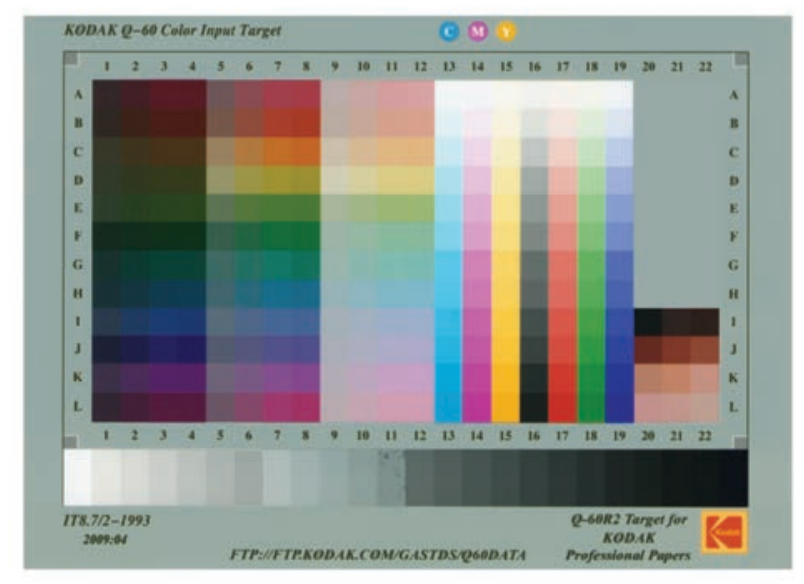

Figure 1. Images of the $\mathrm{X}$-rite ColourChecher ${ }^{\circledR}$ White balance Card (A) and the reference target Kodak Q-60R2 reflection chart (B) used in the calibration process. 
cular portions of fruit skin were acquired through the CVS, and successively with the colorimeter. In this last case, 5 measurements for each circular portion were taken; cause the little measurement area of the colorimeter $\left(3 \mathrm{~mm}^{2}\right)$. The mean of the 5 measurements was considered. The acquiring modalities were the same described in the paragraph 2.3. In total a data set of 12 RGB measurements and their corresponding $\mathrm{L}^{*} \mathrm{a} \mathrm{b}^{*}$ measurements were obtained from the CVS and the colorimeter respectively. The RGB data set was converted in $\mathrm{L}^{*} \mathrm{a}^{*} \mathrm{~b}^{*}$ values using the best of two kind (MFNN and LS-SVM) of trained model.

\section{Least squares support vector machines models}

The LS-SVM regression can be expressed as (Liu et al., 2009):

$$
y(x)=\sum_{k=1}^{N} \alpha_{k} K\left(x, x_{k}\right)+b
$$

where $\mathrm{K}\left(\mathrm{x}, \mathrm{x}_{\mathrm{k}}\right)$ is the kernel function, $\alpha_{\mathrm{k}}$ is the Lagrange multiplier called support value, $\mathrm{b}$ is the bias term. Currently, there is no systematic methodology for selection of kernel function. However, compared with other feasible kernel functions, radial basis function (RBF) as a non linear function is a more compacted supported function kernel and able to reduce the computational complexity of the training procedure and give good performance under general smoothness assumptions (Liu et al., 2009). Thus, RBF kernel was adopted as the kernel function of the LS-SVM models in this study. It can be expressed as follows:

$$
K\left(x, x_{k}\right)=\frac{\exp \left(-\left\|x-x_{k}\right\|\right)^{2}}{2 \sigma^{2}}
$$

whereas $\sigma^{2}$, the squared variance of the Gaussian function, is the kernel parameter. To achieve high level of performance with LS-SVM models, two parameters have to be tuned, the regularization parameter $\gamma$ and the kernel parameter $\sigma^{2}$. Choosing an appropriate regularization parameter and the kernel parameter is an important task and mostly depend on the realized application type. The regularization parameter $\gamma$ determines the trade-off between structural risk minimization principle and empirical risk minimization, and is important to improve the generalization performance of LS-SVM model. The kernel parameter $\sigma^{2}$ controls the value of function regression error, and influences directly the number of initial eigenvalues/eigenvectors. Small values of $\sigma^{2}$ yield a large number of regressors and eventually it can lead to over-fitting. On the contrary, a large value of $\sigma^{2}$ can lead to a reduced number of regressors, making the model simpler, but eventually not so accurate.

Therefore, an efficient search strategy is needed to tune $\gamma$ and $\sigma^{2}$. In this study, we employ a two-stage grid search process on the parameters space. For this purpose, a coarse grid search process is firstly employed to narrow down the search region of the parameters space. In coarse search process, the incremental steps of grid are considerable big to obtain an enough search space. For each grid points, the mean square error (MSE) from L-fold cross validation is determined and minimum MSE interval is detected. In L-fold cross-validation, the trained data is randomly split into L roughly equal subsets. An LS-SVM model is trained using (L-1) of those subsets and validated on the subset left out. This procedure is repeated $\mathrm{L}$ times with each of the $\mathrm{L}$ subsets used as the validation subset in turn. Averaging the validation errors over the $\mathrm{L}$ trials gives a prediction of the generalization error. Then the search is tuned to a finer search in the region where the predicted MSE value from the L-fold cross-validation is the lowest in the coarse search. The minimum MSE value indicates the optimum LS-SVM parameters.
All the calculations were performed using MATLAB v7.0 (The MathWorks). The free LS-SVM toolbox (LS-SVM v1.5, Sukens, Leuven, Belgium) was applied with MATLAB to develop the LS-SVM models.

$\mathbf{A}$

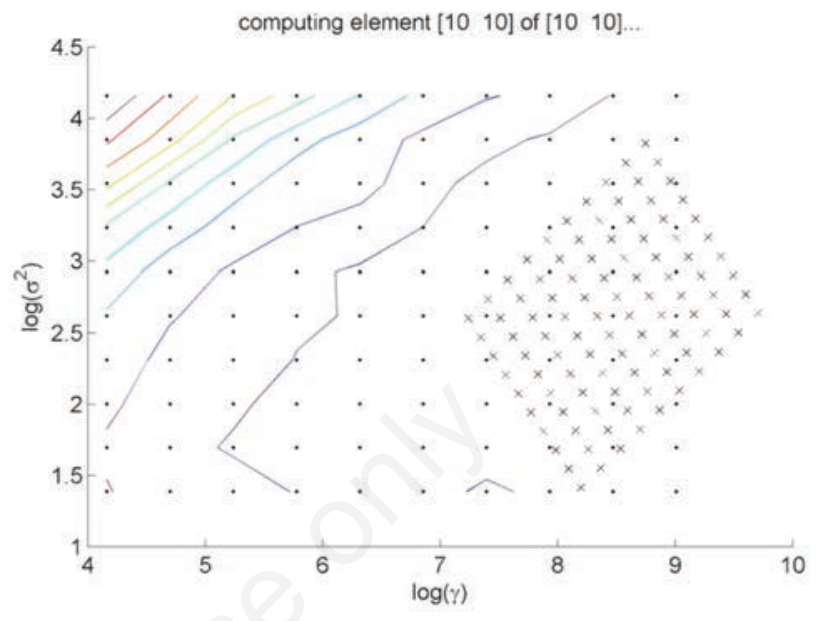

B

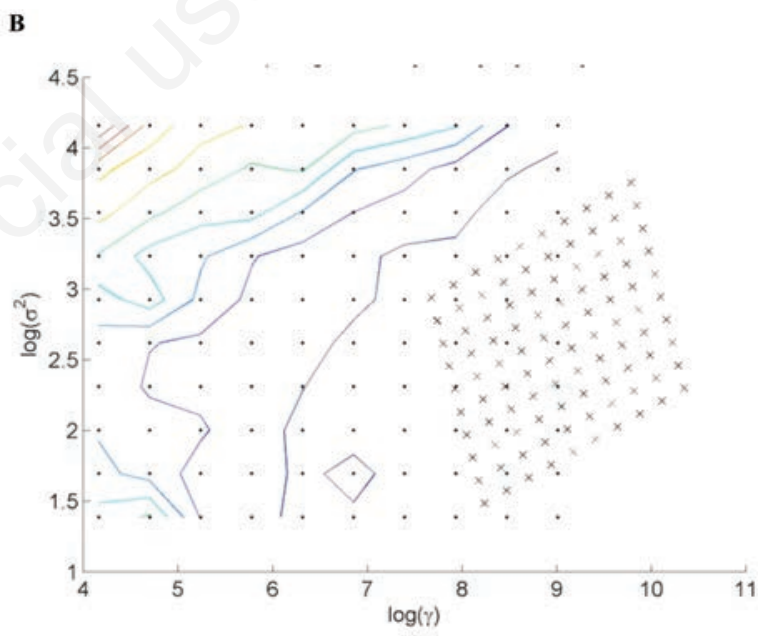

C

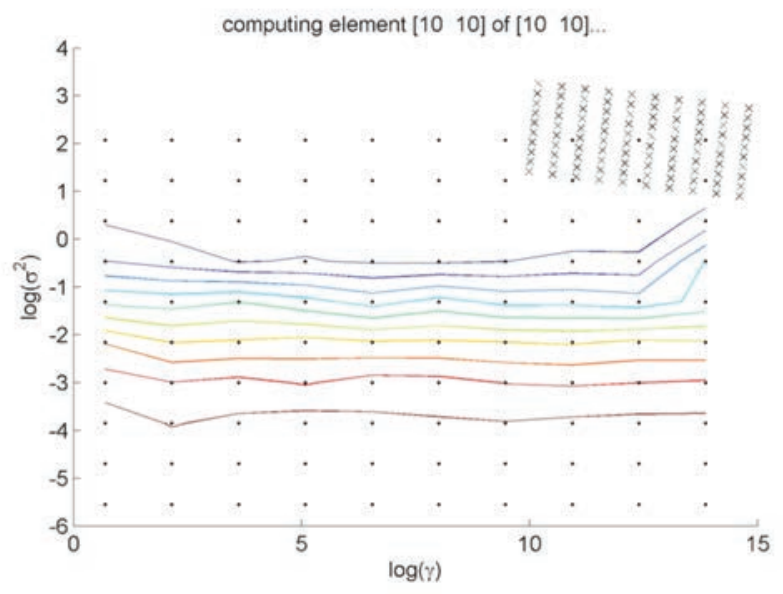

Figure 2. Grid search on $\gamma$ and $\sigma^{2}$ for the prediction of $L^{*}$ values $(A), a^{*}$ values $(B)$ and $b^{*}$ values $(C)$. 


\section{Performance evaluation}

The statistics used for estimating the performance of the regression models developed by LS-SVM and MFNN included determination coefficients for the validation set $\left(\mathrm{R}^{2}\right)$ and root mean square error (RMSE) for the validation set. Moreover the average and standard deviation of colour differences between the $\mathrm{L}^{*} \mathrm{a}^{*} \mathrm{~b}^{*}$ values predicted from the measured RGB values and the $\mathrm{L}^{*} \mathrm{a}^{*} \mathrm{~b} *$ values measured with the colorimeter for the validation set were computed. The colour differences $\Delta \mathrm{E}_{\mathrm{ab}}$ were computed by the following equation:

$$
\Delta E_{a b}=\sqrt{\left(\Delta L^{*}\right)^{2}+\left(\Delta a^{*}\right)^{2}+\left(\Delta b^{*}\right)^{2}}
$$

\section{Results and discussion}

In this study the kernel parameters were optimised for each output variables with values of $\gamma$ in the range of $2^{6}-2^{13}$ and $\sigma^{2}$ in the range of $2^{2}-2^{6}$ with adequate increments. These ranges were chosen from previous studies where the magnitude of parameters to be optimised was established. For each combination of $\gamma$ and $\sigma^{2}$ parameters, the mean square error of 5 -fold cross validation was calculated and the optimum parameters were selected when produced smaller MSE. The optimising process for $\mathrm{L}^{*}, \mathrm{a}^{*}$, and $\mathrm{b}^{*}$ was shown in Figures $2 \mathrm{~A}, \mathrm{~B}$, and $\mathrm{C}$, respectively. The grid '.' in the first step is $10 \times 10$, and the searching step in the first step is large. The optimum search area is determined by error contour line. The grid ' $x$ ' in the second step is $10 \times 10$, and the searching step in the second step is smaller. The optimal search area is determined based on the first step. The optimal pair of $\left(\gamma, \sigma^{2}\right)$ was found at the value of $\gamma=6098.31$ and $\sigma^{2}=12.05$ for $L^{*}$, at the value of $\gamma=$ 15420.61 and $\sigma^{2}=7.59$ for $\mathrm{a}^{*}$, and at the value of $\gamma=203377.21$ and $\sigma^{2}$ $=16.83$ for $\mathrm{b}^{*}$.

The high $\mathrm{R}^{2}$ values, equal to 0.9989 for $\mathrm{L}^{*}, 0.9987$ for $\mathrm{a}^{*}$, and 0.9994 for $b^{*}$, with low RMSE values of $0.6443,0.3226$, and 0.2702 respectively, showed that LS-SVM has strong ability for regression analysis (Figure 3 ). Moreover, the low average $\Delta \mathrm{E}_{\mathrm{ab}}$ of $0.8214 \pm 0.5443$ units showed that the LS-SVM approach provides consistent and accurate results in camera characterization.

In Figure 4 are shown the fittings of measured and predicted values of $\mathrm{L}^{*}, \mathrm{a}^{*}$ and $\mathrm{b}^{*}$ using the MFNN model.

The $\mathrm{R}^{2}$ values resulted equal to 0.9918 for $\mathrm{L}^{*}, 0.9933$ for $\mathrm{a}^{*}$ and $0.9958 \mathrm{~b}$ * but the respective RMSE values of $1.7458,1.2742$, and 1.4144 , resulted higher than those obtained using LS-SVM, showing that MFNN has less ability than LS-SVM for regression analysis.

Figure 5 shows the $\Delta \mathrm{E}$ values calculated on validation set, using the LS-SVM model and a MFNN. As shown in the graph, the LS-SVM model provides a higher accuracy then the MFNN. In fact the average $\Delta \mathrm{E}$ is equal to $0.8232 \pm 0.5033$ for LS-SVM model and $1.7197 \pm 1.6846$ for the MFNN.

The peaks shown in MFNN $\triangle \mathrm{E}$ line indicates that the MFNN has less generalization ability then the LS-SVM model.

The experimental evidences demonstrate that the LS-SVM model performances are higher than MFNN model. Then, the applicative case of fruit colour measurement by CVS was carried out using the trained LS-SVM models. In Figure 6 are shown 4 of the 12 images acquired by the CVS, each one corresponding to a circular skin portion of the four species of fruit considered.

In Figures 7 are shown the fittings of measured and predicted values of $\mathrm{L}^{*}, \mathrm{a}^{*}$ and $\mathrm{b}^{*}$ relieved on skins of fruits, by means of the colorimeter and of the CVS. The RGB data measured by CVS were con-
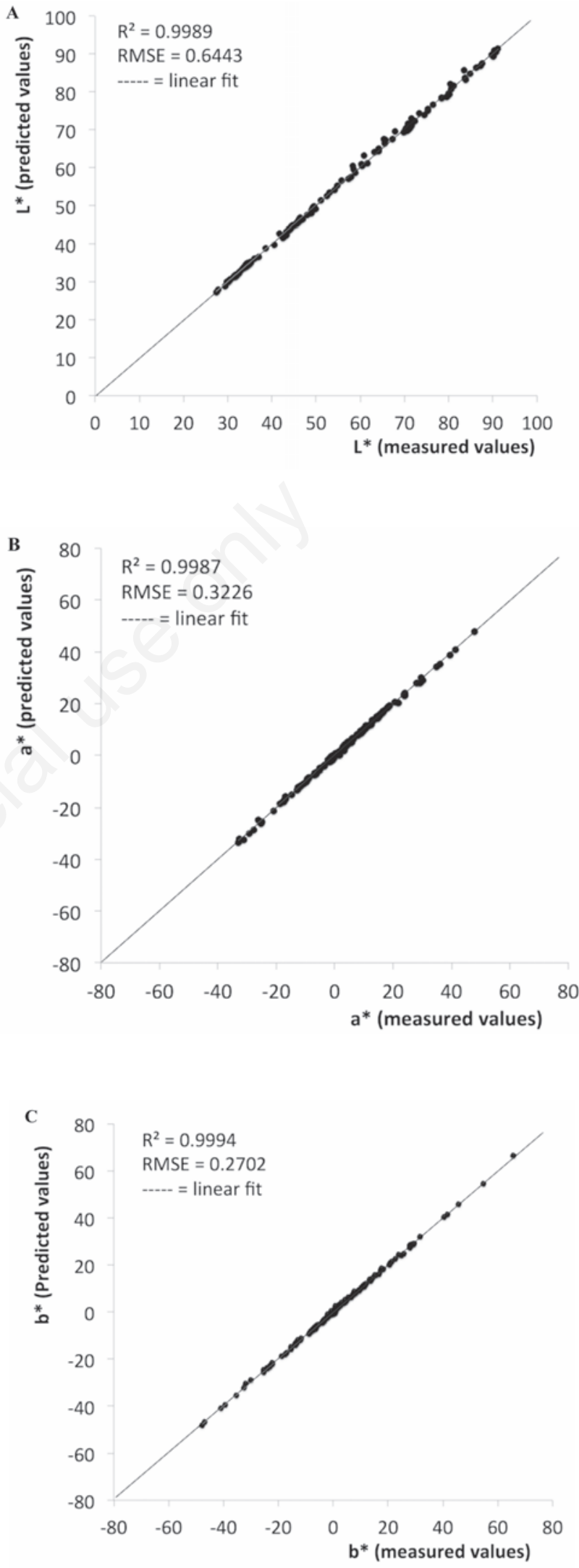

Figure 3. Least squares support vector machine (LS-SVM) predicted $v s$ measured values of $L^{*}(A), a^{*}(B)$ and $b^{*}(C)$ for the validation set. RMSE, root mean square error. 

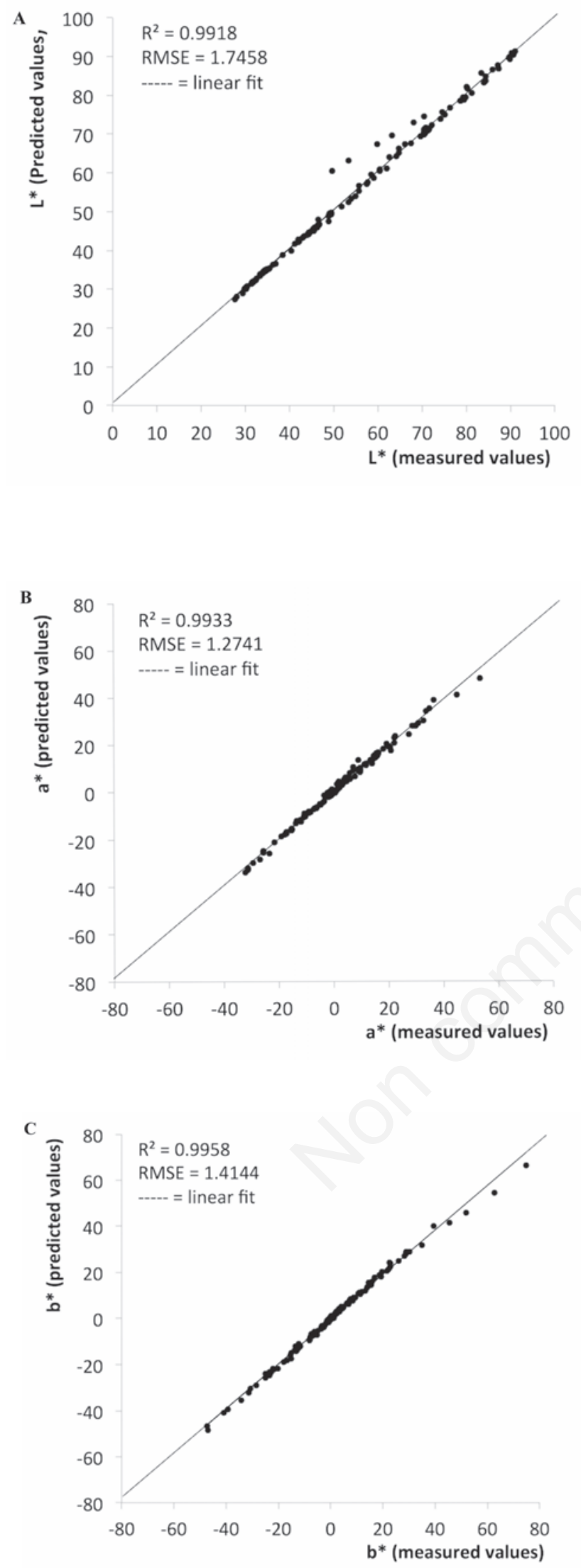

Figure 4. Multilayer feed-forward neural network (MFNN) predicted $v$ s measured values of $\mathrm{L}^{*}(\mathrm{~A}), \mathrm{a}^{*}(\mathrm{~B})$ and $\mathrm{b}^{*}(\mathrm{C})$ for the validation set. RMSE, root mean square error. verted in $\mathrm{L}^{*} \mathrm{a}^{*} \mathrm{~b}^{*}$ values, using the trained LS-SVM models.

Analysing Figure 7, it is possible to notice the high correlations between measured and predicted values. The $\mathrm{R}^{2}$ values resulted equal to 0.9987 for $\mathrm{L}^{*}, 0.9994$ for $\mathrm{a}^{*}$ and 0.9990 for $\mathrm{b}^{*}$. Moreover, the RMSE values resulted equal to 0.2890 for $\mathrm{L}^{*}, 0.3018$ for $\mathrm{a}^{*}$ and 0.3390 for $\mathrm{b}^{*}$. These results confirm that the LS-SVM models works well to predict $\mathrm{L}^{*} \mathrm{a}^{*} \mathrm{~b}^{*}$ values from RGB data of a CDC. Therefore, it is possible to conduct precise and accurate mensuration of $\mathrm{L}^{*}, \mathrm{a}^{*}$ and $\mathrm{b}^{*}$ values of fruits and vegetables by a low cost CVS, with the same precision of a punctual colorimeter.

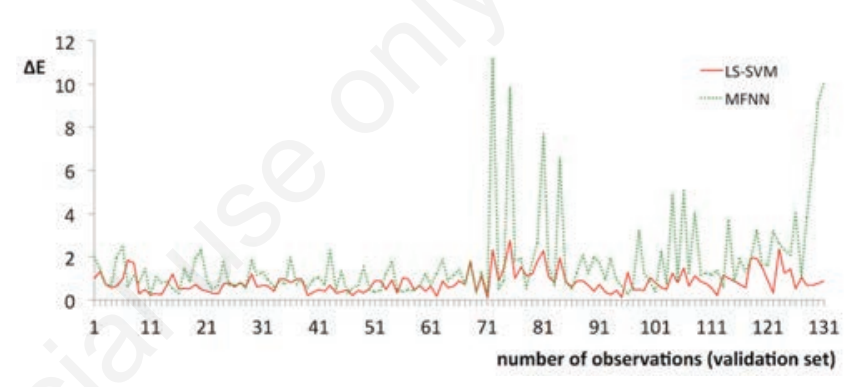

Figure 5. Performance comparison between least squares support vector machine (LS-SVM) model and a 3-8-3 multilayer feed-forward neural network (MFNN).

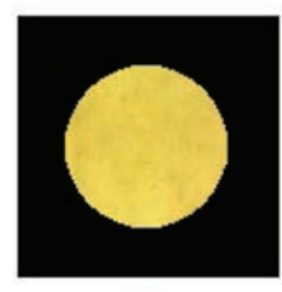

$\mathbf{a}$

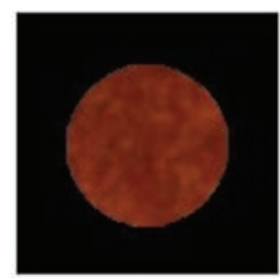

C

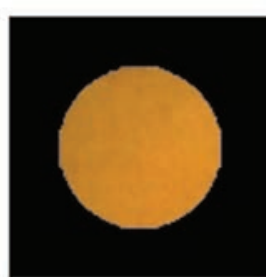

b

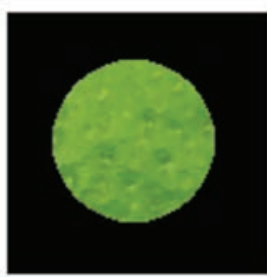

d
Figure 6. Examples of segmented images of circular portion of fruit skins. 

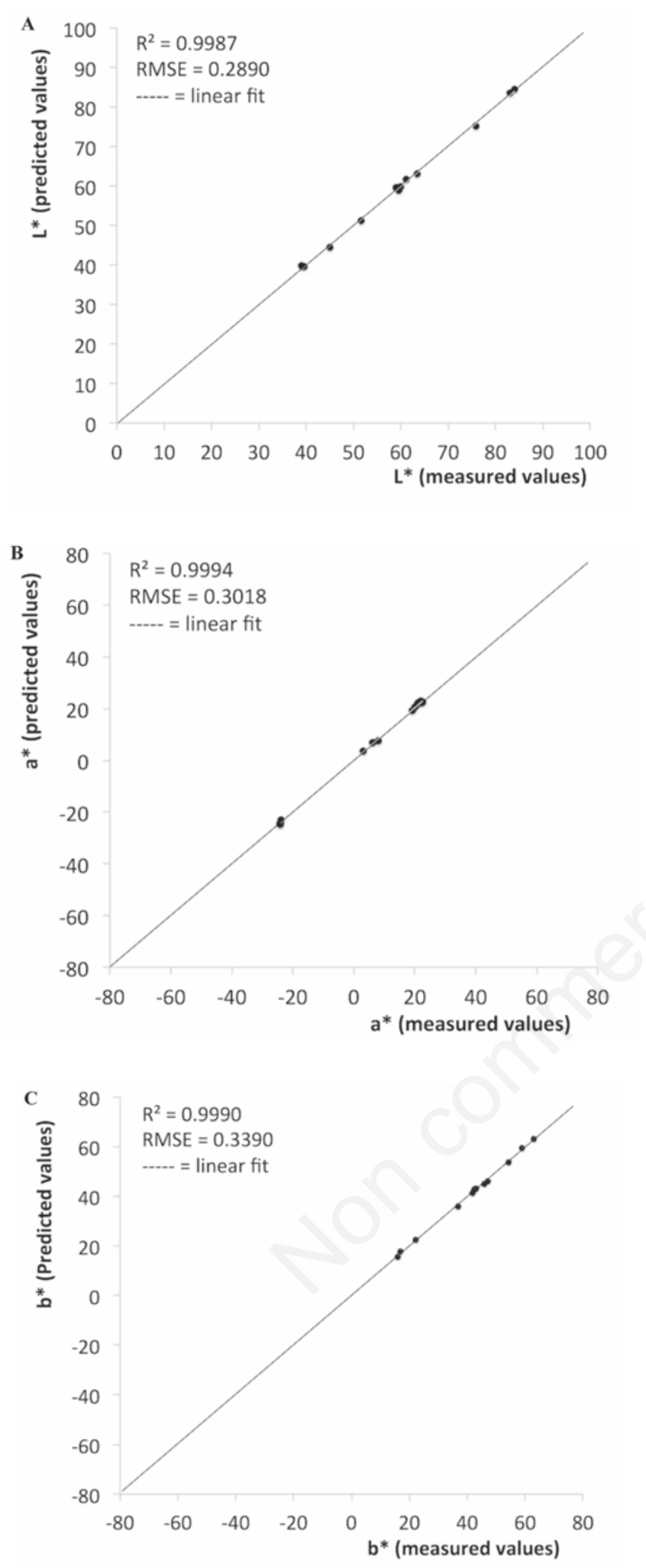

Figure 7. Least squares support vector machine (LS-SVM) predicted $v s$ measured values of $L^{*}(A), a^{*}(B)$ and $b^{*}(C)$ for the data relieved on skins of fruits. RMSE, root mean square error.

\section{Conclusions}

The objective was to evaluate the potential of least squares support vector machine (LS-SVM) regression to develop an efficient method to measure the colour of food materials in $\mathrm{L}^{*} \mathrm{a}^{*} \mathrm{~b}^{*}$ units by means of a CVS. The results of this study shown that this approach produced high precision and accuracy in predicting the $\mathrm{L}^{*} \mathrm{a} * \mathrm{~b}^{*}$ values from the RGB values generated by a CDC. This consideration was reinforced by the measurements carried out on the skins of fruits with different chromaticity. In fact the results of this applicative case shown that the LSSVM regression works well to predict $\mathrm{L}^{*} \mathrm{a}^{*} \mathrm{~b}^{*}$ data from RGB data acquired by a CDC. Moreover, the LS-SVM models shown a higher generalization capability then the MFNN, as confirmed by the $\Delta \mathrm{E}$ values registered on the validation set using the two approaches studied. Therefore, LS-SVM regression appear to be an useful tool to use computer vision systems for high-resolution $\mathrm{L}^{*} \mathrm{a}^{*} \mathrm{~b}^{*}$ colour measurements of food products and any surface, by coupling the precision in the measurement of the colour produced by the colorimeter and the flexibility of processing spatial information that are the prerogative of the CVS.

\section{References}

Hong G., Luo M.R., Rhodes P.A. 2001. A study of digital camera colourimetric characterization based on polynomial modeling. Colour Res. Appl. 26:76-84.

León K., Mery D., Pedreschi F., León J. 2006. Colour measurement in $\mathrm{L}^{*} \mathrm{a}^{*} \mathrm{~b}^{*}$ units from RGB images. Food Res. Int. 39:1084-91.

Liu F., Ye X., He Y., Wang L. 2009. Application of visible/near infrared spectroscopy and chemometric calibrations for variety discrimination of instant milk teas. J. Food Engine. 93:127-33.

Mendoza F., Dejmek P., Aguilera J.M. 2006. Calibrated colour measurements of agricultural foods using image analysis. Postharv. Biol. Technol. 41:285-95.

Mizushima A., Lu R. 2013. An image segmentation method for apple sorting and grading using support vector machine and Otsu's method. Comput. Electr. Agric. 94:29-37.

Sharifzadeh S., Clemmensen L.H., Borggaard C., Støier S., Ersbøll B.K. 2014. Supervised feature selection for linear and non-linear regression of $\mathrm{L}^{*} \mathrm{a}^{*} \mathrm{~b}^{*}$ colour from multispectral images of meat. Engine. Appl. Artif. Intell. 27:211-27.

Suykens J., Vanderwalle J. 1999. Least squares support vector machines. Neural Proc. Lett. 3:293-300.

Valous N.A., Mendoza F., Sun D.W., Allen P. 2009. Colour calibration of a laboratory computer vision system for quality evaluation of presliced hams. Meat Sci. 81:132-41.

Vapnik V. 2000. The nature of statistical learning theory. SpringerVerlag, Berlin, Germany.

Wang D., Wang M., Qiao X. 2009. Support vector machines regression and modelling of greenhouse environment. Comput. Electr. Agric. 66:46-52.

Westland S., Cheung V., Connah D., Ripamonti C. 2004. A comparative study of the characterization of colour cameras by means of neural networks and polynomial transforms. Colour. Technol. 120:19-25. 